\title{
Data Analysis on Medical Service Utilization and Out-of-pocket Spending among Near-poor National Health Insurance Members in South Korea
}

sooyeol park ( $\square$ themia1993@gmail.com )

Seoul National University

Research

Keywords: medical utilization, out-of-pocket spending, catastrophic health expenditure, poverty, relative poverty

Posted Date: December 1st, 2020

DOI: https://doi.org/10.21203/rs.3.rs-113779/v1

License: (c) (1) This work is licensed under a Creative Commons Attribution 4.0 International License.

Read Full License 
1 Data analysis on medical service utilization and out-of-pocket spending

\section{among near-poor National Health insurance members in South Korea}

\section{Sooyeol Park ${ }^{1^{*}}$}

${ }^{1}$ Division of Health Care Management and Policy, Department of Public Health Sciences, Graduate

School of Public Health, Seoul National University, Seoul, Republic of Korea, 08826

\section{* Corresponding author}


Abstract

Background: The near poor, a low income population which is excluded from the Republic of Korea's Medical Aid (MA) program, experiences insufficient use of medical services and high out-of-pocket (OOP) spending due to insufficient coverage by the country's National Health Insurance (NHI). This study aims to examine medical utilization, OOP spending, and occurrence of catastrophic health expenditures (CHE) among the near poor compared to MA beneficiaries and other NHI members.

Methods: A cross-sectional study was conducted drawing upon a nationally representative dataset based on the 2018 Korea Welfare Panel Study (KOWEPS). We classified people into MA beneficiaries, the near-poor population below $50 \%$ of the median income threshold, and other NHI members above the 50\% median income threshold. Using propensity score matching between MA beneficiaries and the near poor and between the near-poor group and the group of those above the poverty line, this study examined medical utilization, OOP spending, and the occurrence of CHE among the study groups.

Results: The findings suggest that MA beneficiaries make greater use of outpatient services compared to the near poor, but other uses of medical services were not significantly different among the study groups. However, OOP spending and occurrence of CHE were significantly higher in the near-poor group compared to the other two groups.

Conclusion: We found that the near-poor group was the most vulnerable group in these terms among the Korean population. Health policy needs to take into account the vulnerability of the near-poor population.

Keywords: medical utilization, out-of-pocket spending, catastrophic health expenditure, poverty, relative poverty 
Universal health coverage aims to provide financial protection from catastrophic health expenditures (CHE) and subsequent impoverishment due to health care costs and also to allow access to essential health services [1]. The South Korean health care system includes the National Health Insurance (NHI) and Medical Aid (MA) programs, both aimed at providing protection from $\mathrm{CHE}$ and ensuring access to essential health services.

Although South Korea achieved a degree of UHC with the establishment of NHI in 1989 , the program has been criticized for insufficient benefit coverage. For example, computed tomography (CT) was not covered until 1995, magnetic resonance imaging (MRI) was excluded from the benefits until 2005 , and overall coverage reached only $62.7 \%$ in 2017 , below the Organization for Economic Cooperation and Development (OECD) average of $73 \%[2,3]$. The original form of MA was initiated in 1997 as a part of the South Korean social welfare program known as the National Basic Livelihood Security System. It was re-envisioned as the MA program in 2001. MA is a public aid program that guarantees access to necessary health services to low-income populations incapable of maintaining their daily lives or who are having difficulty with living costs. It is comparable to the Medicaid program in the US [1]. MA beneficiaries are composed of Type I and Type II recipients based on their level of inability or incapacitation [4]. Type I beneficiaries are exempted from out-of-pocket (OOP) payment for any medical utilization while Type II beneficiaries are assigned minimum copayment rates of up to $15 \%$ [1]. Approximately $3 \%$ of the overall population qualified for MA in 2017 [4].

The near poor are defined under the National Basic Living Security Act as those who are not eligible for public aid programs but who have equivalized disposable household incomes less than $50 \%$ of median ordinary income [5]. The OECD defines relative poverty using the same definition [6]. Because South Korea's entitlement criteria for MA is less than $40 \%$ of median ordinary income and several exceptions exist, South Korea's relative poverty 
rate based on a threshold of below $50 \%$ median ordinary income was $17.5 \%$ in 2017 , but only $3 \%$ of the total population was eligible for MA in that year [7]. The remaining group is enlisted only in NHI, and because of its insufficient coverage, the majority of the low-income population remains in a blind spot within the health care system. With the low coverage provided by South Korea's health insurance, high OOP spending is blamed for the occurrence of unmet needs among the near poor caught in a blind spot in the health care system [8]. Excessive OOP spending among patients with low solvency can result in excessive medical expenses, leaving these populations vulnerable to $\mathrm{CHE}$ and impoverishment due to healthcare expenditures. Because of this under-insuring taking place within NHI, it cannot function properly as a primary social safety net protecting citizens from financial crises caused by illness $[9,10]$. The relatively poor health resulting from their sociodemographic condition means that the near-poor population tends to experience greater medical needs. Due to low coverage under NHI, however, the near poor often cannot use the medical services that they require [11]. Extensive research has been performed on health care utilization and OOP spending among the low-income population in South Korea. Many studies have compared OOP spending and medical utilization among MA beneficiaries and NHI members $[12,13,14]$. The studies reviewed here show that MA beneficiaries tend to use more medical services but experience less OOP spending compared to NHI members. For instance, Kim (2015)[12] found that the number of outpatient visits was 1.431 times higher, and the hospitalized days per year was 1.604 times higher among MA beneficiaries compared to NHI members. However, studies examining health utilization and OOP spending among the near poor are limited. Lee (2016)[8] compared OOP spending and medical utilization among MA beneficiaries and NHI members by using propensity score matching, and because equivalized disposable household income was included in the matching variables, the study population extracted from NHI members had similar characteristics to those defining the near-poor group. Lee (2016)[8] found that a person 
enrolled in MA had less OOP spending for hospitalization and outpatient visits and their number of days of hospitalization was greater compared to NHI members. A study which compared MA benefits and Medicaid among low income populations found that the near poor in South Korea had similar sociodemographic characteristics with MA beneficiaries [5]. Choi (2015)[15] found that poor people not enrolled in MA had significantly lower medical utilization compared to MA beneficiaries, and greater healthcare costs as well. However, these studies have only compared the near poor with MA beneficiaries and do not include the population above the threshold of $50 \%$ of median income, or they did not divide the lowincome groups according to specific criteria to separate the near poor out of the low-income population. Because of the existence of supportive programs for CHE and aid for OOP spending among the near-poor population and because the near poor have distinctive sociodemographic conditions compared to other NHI members above the 50\% income threshold, there is a need to distinguish the near-poor population from other NHI members and compare them to examine the different socio-demographic and policy contexts they inhabit. In addition, studies using propensity score matching or defining the near poor have relied only on income to distinguish the near poor from other NHI members. The reasons for poor people being excluded from MA include not only the income threshold, but other reasons as well [7]. For instance, those whose obligatory provider exceeds certain criteria are excluded from MA enrollment.

Therefore, this study examines general characteristics by dividing total respondents by equivalized disposable household income of $50 \%$, and then separates the near-poor population from the low-income population according to specific criteria which will be discussed later. In addition, we analyzed medical utilization and OOP spending among three separate groups.

\section{Material and methods}




\section{Data source}

We collected individual data from the $14^{\text {th }}$ Korea Welfare Panel Study (2019) database, which is conducted by Seoul National University (SNU) and the KIHASA. The Korea Welfare Panel Study was designed to provide a probability sample of South Korea's population. The study subjects were selected to compare low-income and general families by collecting half of the samples from low-income households [16]. Data collected from February 18 through May 21, 2019 were used for this study. The period of the survey was January 1 through December 31, 2018 for flow data and December 31, 2018 for stock data.

Among the total of 14,418 individuals and 6,331 households initially selected for this study, 3,183 individuals were excluded due to being a minor under the age of 18 , missing health care program type information, being beneficiaries of free medical treatment for reasons of national merit, and as individuals above the poverty line but still beneficiaries of MA. Eventually, 11,235 individuals were selected as subjects of this study

\section{Variable definition}

\section{Defining the Study Group}

The study population was categorized into three groups: MA beneficiaries, the near poor, and those above the poverty line. The poverty line was defined as $50 \%$ of median income by the number of household members in 2018. Near poor was defined as the population who are below the poverty line and enrolled in NHI, or who were subject to national basic living security aid in 2018 but not enlisted in MA because the obligatory provider's income or property exceeded criteria or for a failure to pay the NHI contribution for more than six months and thus being excluded from NHI benefits. NHI members not grouped as near poor were 
placed in the above-poverty-line group. The entire population enlisted as MA beneficiaries were grouped as MA beneficiaries.

\section{Selection and definition of explanatory variables}

We examined two types of medical utilization for 2018: outpatient services and inpatient services. Inpatient services were examined in terms of three variables: hospital visits, hospitalized days, and hospitalized days per visit. For OOP spending, the Korea Welfare Panel Study includes all OOP spending, including hospital costs, dental costs, Korean traditional medicine costs, and drug costs. CHEs are defined as annual OOP spending exceeding a specified fraction of annual income, which is distinct from high health costs defined simply as those exceeding a determined amount $[17,18]$. The specified fraction threshold varies between $10 \%$ to $40 \%$; for this study, $20 \%$ and $40 \%$ were used as a threshold.

Sex, age, marital status, education, employment, income, self-reported health status, chronic disease states, private insurance coverage status, depression, and other disorders were set as predisposing factors affecting medical utilization and OOP spending. Educational achievement was grouped into no completion, below high school diploma, high school diploma, and above high school diploma. Employment was grouped into temporary employee; employer, self-employed, pr unpaid family worker; unemployed or economically inactive; and permanent employee. Income was defined as equivalized disposable personal income by adding gross income and non-consumption expenditures to calculate household disposable income and dividing household disposable income by the square root of the number of household members. Self-reported health status was measured with "very healthy," "healthy," "moderate" deemed healthy and "not very healthy" and "unhealthy" as bad health. Private insurance coverage status was measured by whether the respondent has at least one type of private insurance. Depression status was measured on the CESD-11 scale. Depression status was defined as the sum of 
CESD-11 questionaries ( 0 - 33 points) multiplied by $20 / 11$ being greater than or equal to 16 [19]. Respondents were grouped as having another disorder if any type of mental, kidney, heart, respiratory, liver, physical, speech, facial nerve, brain lesion, visual disturbance, hearing impairment, mental retardation, or intestinal disorder was present.

\section{Statistical analyses}

We examined the effect of MA and poverty on health utilization and OOP spending. Because the decision to use medical services and incur OOP spending is not random given that an individual's health status, occupational status, and various other factors influence it, we applied the model by Rubin (1974)[20]. Following his notation we observed $Y=\alpha+\beta \cdot X+$ $\delta \cdot T+\epsilon$, where $\mathrm{T}$ is a 0 to 1 indicator of whether an individual is assigned to the treatment (MA beneficiary or below poverty line) or control group, $\mathrm{X}$ is the observable factors, $\mathrm{Y}$ is the outcome (medical utilization, OOP spending, or occurrence of CHEs), and $\epsilon$ is unobservable but influences Y. If we simply compare the realized outcomes, for instance, based on the average treatment effect on the treated (ATT), that is $E\left(Y_{1} \mid T=1\right)-E\left(Y_{1} \mid T=0\right)$, selection bias will occur due to the non-randomness of factors that influence the decision [21]. However, if the assignment of treatment is random for individuals with similar values of observable covariates, the ATT can be identified.

We used propensity score matching to estimate treatment's effects in an unbiased manner by accounting for possible covariates that predict receiving treatment [22]. To conduct matching between the MA and near-poor groups, a propensity score was derived by applying a probit model, setting policy variables as to whether the individual is an MA beneficiary, and other covariates were adjusted between the control and case group. Sex, age, marital status, education, employment, income, self-reported health status, chronic disease states, private 
insurance coverage status, depression, and other disorders were set as covariates.

Propensity score for respondent $i$ is the conditional probability of assignment to the treatment condition, as follows.

$$
\begin{gathered}
\operatorname{Pr}\left(T_{i}=\text { medical aid, poverty line }\right)=\frac{e^{X \beta}}{1+e^{X \beta}}, \\
\text { where } \ln \left(\frac{p}{1-p}\right)=\beta_{0}+\beta_{1} X_{1}+\cdots \beta_{k} X_{k}
\end{gathered}
$$

group. Given that an increasing number of controls matched to each case resulted in improved efficiency, but efficiency is minor when one-to-M matching exceeds $\mathrm{M}=5$, we applied one-tofour nearest neighbor matching with replacement and 0.01 caliper width [23]. After matching was completed, a t-test was applied to verify whether the covariates' distribution was the same between the groups.

To conduct matching between the near-poor and above-poverty line groups, the unmatched near-poor groups with MA beneficiaries were excluded. The rest of the near-poor sample was matched with the above-poverty line group, setting policy variables as whether or not the individual is below the poverty line. The same procedure was conducted to derive the propensity score and matching between the near-poor and above-poverty-line groups.

After matching was completed, regression was applied to check each policy variable's effect on medical utilization and OOP spending. We assumed that the outpatient and inpatient medical usage in this analysis follows a Poisson basic model [24]. For medical utilization, there are three fundamental statistical properties: 1) To be non-negative; 2) to have no nontrivial fraction of zero outcomes; 3) to follow a positively skewed distribution of non-zero realization [25]. To accommodate these unique count data structures, a zero-inflated Poisson 
(ZIP) model was used to model hospital visits, hospitalized days, and hospitalized days per visit. For outpatient use, we applied a negative binomial model.

Let $x_{i}$ be the vector for the covariates with $\mu_{i}$ the expected number of occurrences where $x_{i}$ is the vector independent variable and $\beta$ the vector of parameters to be estimated.

$$
\mu_{i}=\exp \left(\beta_{0}+\beta_{1} x_{1 i}+\beta_{2} x_{2 i}+\cdots+\beta_{k} x_{k i}\right)=\exp \left(x_{i}^{\prime} \beta\right)>0
$$

The ZIP model first models the probability of observing zeroes using logistic regression, and then uses a Poisson regression to model the non-zero count data while accounting for the excess zeroes. The ZIP model can be presented as $y_{i}$, which represents the count of the hospital visits, hospitalized days, and hospitalized days per visit for the $i$ th person and $\pi$ as the probability of a case in which the count is zero and $1-\pi$ is the probability of a case in which the count is not zero. Therefore, the probability distribution of $y_{i}$ can be written as follows:

$$
\operatorname{Pr}\left(y_{i}=j\right)=\left\{\begin{array}{l}
\pi_{i}+\left(1-\pi_{i}\right) \exp \left(-\mu_{i}\right) \text { if } j=0 \\
\left(1-\pi_{i}\right) \frac{\mu_{i}^{y_{i}} \exp \left(-\mu_{i}\right)}{y_{i} !} \text { if } j>0
\end{array}\right.
$$

The negative binomial model can be written as below, where $y_{i}$ presents the count of the dependent variable outpatient use for the $i$ th person and $\alpha=\frac{1}{v}$, where $v$ is the scale parameter of the gamma noise variable, and the negative binomial regression model can be shown as follows:

$$
\operatorname{Pr}\left(Y=y_{i} \mid \mu_{i}, \alpha\right)=\frac{\Gamma\left(y_{i}+\alpha^{-1}\right)}{\Gamma\left(\alpha^{-1}\right) \Gamma\left(y_{i}+1\right)}\left(\frac{1}{1+\alpha \mu_{i}}\right)^{\alpha^{-1}}\left(\frac{\alpha \mu_{i}}{1+\alpha \mu_{i}}\right)^{y_{i}}
$$

Because the OOP spending data is skewed to the right and was not normally distributed, 
log-link Generalized Linear Model(GLM) was applied to model OOP spending [26]. The gamma distribution is undefined for values of ' 0 ', an offset of 0.00001 was added to each OOP spending value in consideration of the users who had no OOP spending, and results were interpreted with exponentiated coefficients [27].

The occurrence of CHEs was modeled by applying binomial logistic regression to estimate each group's risk ratio and risk difference. All statistical analyses were performed using Stata ver. 16 (StataCorp, College Station, Texas, USA). This study protocol was approved by the Institutional Review Board of Seoul National University (IRB No. E2010/001004).

\section{Results}

\section{Demographic characteristics}

Various demographic and socio-economic characteristics, health status, and occupation status among three study groups are compared (Table 1). MA beneficiaries and the near-poor group were found to show similar health and socio-economic status. However, the near-poor group tends to be more economically active than the MA beneficiaries. The abovepoverty-line group was found to have better health and socioeconomic status and was more economically active.

\section{(Table 1 here)}

\section{Matching quality}

Overall descriptive statistics for all covariates were compared among the three study 
groups. Before matching, almost all covariates are statistically different between the groups at the 5\% significance level. After matching, there are no significant differences in all covariates among the groups. A matched sample of 507 MA beneficiaries and 915 individuals from the near-poor group was generated and used in the subsequent analysis. For the near-poor and above-poverty line group, five of the 915 in the matched sample for the MA and near-poor groups were excluded because common support was not achieved. A matched sample of 910 from the near-poor group and 1,482 from the above-poverty-line group were generated and used in the subsequent analysis.

\section{(Table 2 here)}

\section{Differences in health utilization and OOP spending among Medical Aid beneficiaries and} the near-poor group

The MA beneficiaries group was found to use $35 \%$ more outpatient services than the near-poor group $(p<0.001 ; \exp (0.30)=1.35)$. The two groups showed no significant difference in hospital visit frequency and hospitalized days per visit. The MA group showed a significantly lower number of expected hospitalized days than the near-poor group. The expected number of hospitalized days for the MA group was estimated to be $31 \%$ smaller than the near-poor group $(p<0.1 ; \exp (-0.36)=0.69)$. The other three occupation groups were found to use significantly less use outpatient services and hospital visits than permanent employees at the at least $10 \%$ significance level. Private insurance subscription had a negative effect on hospital days and hospital days per visit based on Poisson estimation $(p<0.05)$ (Table 3). 
OOP spending and CHE occurrence were compared among the near poor and MA

\section{(Table 4 here)}

beneficiaries. MA beneficiaries showed $73.6 \%$ lower OOP spending and less chance of CHE experience for both the $20 \%$ and $40 \%$ thresholds compared to the near-poor group $(p<0.001)$ (Table 4). Permanent job status was found to show a lower chance of occurrence of CHE than the other occupational statuses, with the exception of unemployed or economically inactive (at least $10 \%$ significance level). People with private insurance responded as spending $41.9 \%$ more on OOP spending $(p<0.05)$, but had no significant effect on occurrence of CHE.

\section{Differences in medical utilization and OOP spending among the near-poor and above-} poverty-line groups

There was no significant difference in medical utilization except hospitalized days and hospitalized days per visit between the near-poor and above-poverty-line groups. For hospitalized days, the higher use of hospitalized days was conditional on being a hospital user, as Poisson and logit estimates were both positive $(p<0.1)$. The near-poor group had a $23 \%$ greater chance of no hospital visits $(p<0.1 ; \exp (0.21)=1.23)$. Occupation status was not a significant factor influencing medical utilization. Private insurance subscription was found to be negatively associated with the chance of no hospital visits, higher number of hospital visits, and hospitalized days at the at least $10 \%$ significance level. 8 

compared to the above-poverty-line group (Table 6). Respondents with a permanent job had less chance of experiencing CHEs at the $40 \%$ threshold compared to any other occupational status, except unemployed or economically inactive.

\section{(Table 6 here)}

\section{Discussion}

We found that the near-poor group was the most vulnerable group among the Korean population. There were no significant differences in medical utilization between the near-poor and above-poverty-line group except hospital days after controlling for potential bias and between the near-poor group and MA beneficiaries except outpatient use. In addition, the nearpoor group was found to make more OOP spending by $73.55 \%$ than did MA beneficiaries and to have significantly higher chance of experiencing CHE at both the $40 \%$ and $20 \%$ thresholds compared to the two other groups after equivalized disposable personal income was adjusted. These results demonstrate that the near-poor group could be the most vulnerable population based on medical service utilization and OOP spending. This assumption is in agreement with the results reported by several other studies. MA beneficiaries were found to use more inpatient and outpatient services, incurred less OOP spending, and had a lower chance of experiencing CHEs compared to NHI members $[8,28]$. A study which defined the near poor as people not enrolled in MA with income less than $120 \%$ of the minimum cost of living found that MA beneficiaries experienced significantly higher health care utilization in terms of both outpatient visits and inpatient visits, and lower health care costs and proportion of OOP spendings to income compared to the poor not enrolled in MA [15]. However, contrary to previous findings, our study shows that the difference in medical utilization only applies to outpatient visits 
among MA beneficiaries and the near poor. This might demonstrate the effect of recent policy changes. CHE support for the population below $50 \%$ of the median income, which matches the defined near-poor population in this study, was implemented for severe diseases in 2013 and expanded to all diseases in 2018 [29]. Furthermore, the current administration implemented an NHI coverage expansion in 2017 by alleviating uncovered services and restricting the OOP spending threshold to $10 \%$ of annual income for the bottom $50 \%$ income group [30]. Because previous research was based on data from before 2016, the findings do not reflect these recently implemented policies affecting the medical utilization of the near poor. Further study is needed to examine the effects of this recent policy change on medical utilization among the near poor. Also, compared to outpatient services, inpatient services rely more on the decision of health experts than the patient's discretion $[31,32]$. Hospitalization and length of stay is affected more by health experts, commonly physicians, so factors of individual patients may not affected inpatient service to a great degree.

The presence of chronic disease was significantly associated with greater numbers of outpatient and inpatient visits among the three study groups, but were not related to OOP spendings and CHEs beyond the case of the CHE threshold of 20\% among NHI members. Chronic disease has been confirmed in several studies as a significant factor in determining outpatient service use, such as the number of outpatient visits [33]. Because the average age of our study population is high, the presence of chronic disease likely influences higher inpatient use due to a lack of proper self-management $[34,35]$. Additional focus is required on chronic disease prevention by empowering the population through strengthening education. Selfmanagement programs must be supported in order to mitigate hospitalization due to chronic disease. Also, perceived negative health status was associated with a higher volume of outpatient and inpatient use. Perceived negative health status can lead to poor physical health and greater social isolation [36]. Thus, self-evaluated health status must be considered in the 
development of health promotion programs among both MA beneficiaries NHI members. In the comparison of hospitalized days and private health insurance subscription among the near-poor and above-poverty-line groups, the conflict result of Poisson and logit estimators may reflect the difference characteristics of subgroups among the near poor and subscribers to private health insurance [24]. For hospitalized days, it may indicate a subgroup among the near poor which may have both a higher number of hospitalized days overall and a greater probability of no hospital visits. This might indicate the possibility of preventable hospitalization due to a lack of hospital visits. Because of the socio-economic status of the near-poor, it can be presumed that they felt a significant financial burden from hospital visits [37]. Insufficient hospital visits could result in deterioration of health and eventually hospitalization and longer length of stays.For private health insurance subscription, it might indicate that the subgroup of subscribers may have a lower number of hospital visits overall but less probability of no hospital visits.

South Korea has constantly implemented expansions of NHI coverage and pursued the reduction of copayments and support programs for CHEs among the near-poor population to ensure proper health care use and to prevent impoverishment by health care costs. Despite these efforts, several studies, including ours, have demonstrated that the near-poor population still remains unprotected from the occurrence of CHEs. Moreover, previous studies have found that the near-poor population is reported to experience higher unmet needs compared to MA beneficiaries [13. 38]. An expansion of MA could be considered an alternative for alleviating this burden and ensuring essential health services among the near-poor. Lee (2020)[14] found that people who shifted from NHI to MA increased their number of outpatient visits without increasing OOP spending. A more focused policy regarding populations in a blind spot within the health care system, including on perceived health status and chronic disease, is required to ensure essential health services for the near-poor group. 
to other countries with different medical utilization and OOP spending programs. This study conducted a cross-sectional analysis including 516 MA beneficiaries, 915 among the nearpoor, and 1,492 in the above-poverty-line group after matching, which could be an insufficient sample size for analysis using several independent variables. Second, we could not resolve the issue of supply-induced demand or demand-induced demand among MA beneficiaries. Third, although we used propensity score matching to adjust the potential bias, we could not examine several factors that may influence medical utilization and OOP spendings due to a lack of data. Finally, given the limits of the data, we could not identify the use of uncovered medical services because medical services were not categorized as covered or uncovered services. Because several high-quality medical services offered in South Korea are uncovered by either MA or NHI, we cannot verify the quality of medical service that respondents used. Future research should examine various factors that may influence medical utilization and OOP spendings, including variables such as unmet need, health service quality, and service accessibility related factors.

The strengths of this study include its analysis of socio-economic and health-related factors and the use of several statistical methods to accommodate the unique characteristics of outcome variables and minimize potential bias. In addition we examined the medical utilization and OOP spendings of an above-poverty-line group not included in previous studies [39].

\section{Conclusions}

This study found that the near-poor population showed no significant difference in medical utilization compared to the MA and above-poverty-line groups, but that they incurred greater OOP spending and were exposed to a higher chance of experiencing CHEs. This result indicates that the near-poor group is the most vulnerable within South Korea's population. 
411 Health policy needs to take into account this vulnerability of the near-poor population and several factors, such as chronic disease and perceived health status, that significantly influence medial use and cost in order to ensure essential services and provide protection from impoverishment by health care costs.

\section{List of abbreviations}

Medical Aid (MA), out-of-pocket (OOP), National Health Insurance(NHI), Korea Welfare Panel Study (KOWEPS), catastrophic health expenditure (CHE), World Health Organization (WHO), computed tomography (CT), magnetic resonance imaging (MRI), Organization for Economic Cooperation and Development (OECD), Health Insurance Review \& Assessment Service (HIRA), Korea Institute for Health and Social Affairs (KIHASA), Seoul National University (SNU), Center for Epidemiological Studies-Depression (CESD), average treatment effect on the treated (ATT), zero-inflated Poisson (ZIP), Generalized Linear Model (GLM),

\section{Acknowledgments}

I'm grateful to professor Soonman Kwon, Wankyo Chung for his help with the interpretation of the data and professor Juhyun Park, Taejin Lee for their help with methods.

\section{Funding}

There was no external funding for this resaech

\section{Availability of data and materials}

The dataset analyzed during the current study are available in the Korea Welfare Panel Study, [https://www.koweps.re.kr/] 
This study protocol was approved by the Institutional Review Board of Seoul National University (IRB No. E2010/001-004).

440

Competing interests

The author declares that he has no competing interests.

\section{References}

1. World Health Organization (WHO). Regional Office for the Western P. Republic of Korea health system review. Manila : WHO Regional Office for the Western Pacific; 2015.

2. Oh J, Ko Y, Baer Alley A, Kwon S. Participation of the Lay Public in DecisionMaking for Benefit Coverage of National Health Insurance in South Korea. Health Systems \& Reform. 2015;1(1):62-71.

3. Organization for Economic Co-operation and Development (OECD). Health at a Glance 2019: OECD Indicators. Paris: OECD publishing; 2020.

4. National Health Insurance Service (NHIS), Health Insruance Review \& Assessment Service (HIRA). Medical Aid Statistics 2018. NHIS HIRA; 2019.

5. Kim J. Comparative Study on Public Health Care Coverage for Low Income Bracket -Comparison between Medical Benefits and Medicaid-. Korean Comparative Government Review. 2013;17(3):195-220.

6. OECD. Society at a Glance 2019: OECD Social Indicators. Paris: OECD Publishing; 2019. 
$4607 . \quad$ Lee H, Park H, Lee J. Statistical Yearbook of Poverty 2019. In: Korea Institute for Health and Social Affairs (KIHASA). 2019.

8. Lee H. Healthcare utilization and out-of-pocket spending of Medical Aids recipients in South Korea: a propensity score matching with National Health Insurance participants. Korean Health Economic Review. 2016;22(2):29-49.

9. Kim J. Equity in Health Levels and Health Care Utilization of Elderly People in Korea. Social Science Research Review. 2011;27(2):65-87.

10. Kwon S. Thirty years of national health insurance in South Korea: lessons for achieving universal health care coverage. Health Policy Plan. 2009;24(1):63-71.

11. Heo J, Oh J, Kim J, Lee M, Lee J, Kwon S, et al. Poverty in the midst of plenty: unmet needs and distribution of health care resources in South Korea. PloS one. 2012;7(11):e51004-e.

12. Kim J, Lee K, Yoo K, Park E. The differences in health care utilization between Medical Aid and health insurance: a longitudinal study using propensity score matching. PLoS One. 2015;10(3):e0119939.

13. Choi H. Catastrophic health expenditure and Unmet needs of low-income households depending on the types of Healthcare system Seoul National University; 2017.

14. Lee D, Jang J, Choi D, Jang S, Park E. The effect of shifting medical coverage from National Health Insurance to Medical Aid type I and type II on health care utilization and out-of-pocket spending in South Korea. BMC Health Services Research. 2020;20(1):9-79.

15. Choi J, Park E, Chun S, Han K, Han E, Kim T. Health care utilization and costs among medical-aid enrollees, the poor not enrolled in medical-aid, and the near poor in South Korea. Int J Equity Health. 2015;14:128-. 
16. Korean Welfare Panel Study. Korean Welfare Panel Study User Guidelines. In: Korea Institute for Health \& Social Affairs Social Welfare Research Center of Seoul National University. 2020.

17. Wyszewianski L. Financially Catastrophic and High-Cost Cases: Definitions, Distinctions, and Their Implications for Policy Formulation. Inquiry. 1986;23(4):382-94.

18. Wagstaff A. Measuring Financial Protection in Health. Policy Research Working Paper 4554. 2008.

19. Jun J, Yee N. Depression, Its Concurrent Chronic Illnesses, and Related Health Service Use in the Korea Welfare Panel Data. Health and Welfare Policy Forum: KIHASA; 2015. p. 75-84.

20. Rubin DB. Estimating causal effects of treatments in randomized and nonrandomized studies. Journal of Educational Psychology. 1974;66(5):688-701.

21. Schmitz H, Westphal M. Short- and medium-term effects of informal care provision on female caregivers' health. Journal of Health Economics. 2015;42:174-85.

22. Rosenbaum P, Rubin D. The Central Role of the Propensity Score in Observational Studies For Causal Effects. Biometrika. 1983;70:41-55.

23. Austin PC. Statistical Criteria for Selecting the Optimal Number of Untreated Subjects Matched to Each Treated Subject When Using Many-to-One Matching on the Propensity Score. American Journal of Epidemiology. 2010;172(9):1092-7.

24. Solé-Auró A, Guillén M, Crimmins EM. Health care usage among immigrants and native-born elderly populations in eleven European countries: results from SHARE. Eur J Health Econ. 2012;13(6):741-54.

25. Moon S, Shin J. Health care utilization among Medicare-Medicaid dual eligibles: a count data analysis. BMC Public Health. 2006;6:88. 
26. Hill S, Miller G. Health expenditure estimation and functional form: applications of the generalized Gamma and extended estimating equations models. Health economics. 2009;19:608-27.

27. Liao E, Leahy M, Cummins G. The costs of nonsedating antihistamine therapy for allergic rhinitis in managed care: an updated analysis. Am J Manag Care. 2001;7(15 Suppl):S459-68.

28. Kong N, Kim D. Factors influencing health care use by health insurance subscribers and medical aid beneficiaries: a study based on data from the Korea welfare panel study database. BMC Public Health. 2020;20(1):1133.

29. Minisry of Health and Welfare (MOHW). 2020 catastrophic health expenditure support program guidance. MOHW; 2020.

30. Kang H. Issues and Policy Options for Moon Jae-in Care. Health and Welfare Policy Forum: KIHASA; 2018. p. 23-37.

31. Newhouse JP, Manning WG, Keeler EB, Sloss EM. Adjusting capitation rates using objective health measures and prior utilization. Health Care Financ Rev. 1989;10(3):41-54

32. Lee H, Lee T, Jeon B, Jung Y. Factors Related to Health Care Utilization in the Poor and the General Populations. Korean Health Economic Review. 2009;15(1):79-106.

33. Gotsadze G, Tang W, Shengelia N, Zoidze A. Determinants analysis of outpatient service utilisation in Georgia: can the approach help inform benefit package design? Health research policy and systems. 2017;15(1):15-36.

34. Nie JX, Wang L, Tracy CS, Moineddin R, Upshur RE. Health care service utilization among the elderly: findings from the Study to Understand the Chronic Condition Experience of the Elderly and the Disabled (SUCCEED project). J Eval Clin Pract. 2008;14(6):1044-9 
35. Vegda K, Nie JX, Wang L, Tracy CS, Moineddin R, Upshur RE. Trends in health services utilization, medication use, and health conditions among older adults: a $2-$ year retrospective chart review in a primary care practice. BMC Health Serv Res. 2009;9:217.

36. Gunzelmann T, Hinz A, Brähler E. Subjective health in older people. Psychosoc Med. 2006;3:Doc02-Doc.

37. Shin H, Kim S. The Impact of Medicaid Expansion to include population with low income on the preventable hospitalizations. Korean Journal of Health Policy \& Administration. 2010;20(1):87-102.

38. Kim T. The current state and affecting factors of unmet medical needs in Medical Aids recipients and lower income group: Hanyang University; 2020.

39. Kim E. Poverty dynamics, health care utilization, and health expenditure in South Korea: Seoul National University 2019. 